\title{
Towards Understanding the Importance of Co-Located Gameplay
}

\section{Rina R. Wehbe}

HCI Games Group

University of Waterloo

200 University Ave W, N2L3G1

Waterloo, ON, Canada

rina.wehbe@gmail.com

\section{Lennart E. Nacke}

HCI Games Group

University of Waterloo

200 University Ave W, N2L3G1

Waterloo, ON, Canada

lennart.nacke@acm.org
Permission to make digital or hard copies of part or all of this work for personal or classroom use is granted without fee provided that copies are not made or distributed for profit or commercial advantage and that copies bear this notice and the full citation on the first page. Copyrights for third-party components of this work must be honored. For all other uses, contact the Owner/Author.

Copyright is held by the owner/author(s).

CHI PLAY 2015, October 03-07, 2015, London, United Kingdom

c) Lennart Nacke, 2015. This is the author's version of the work. It is posted here for your personal use. Not for redistribution. The definitive version was published in CHI PLAY '15 Proceedings of the 2015 Annual Symposium on Computer-Human Interaction in Play

http://dx.doi.org/10.1145/2793107.2810312

\begin{abstract}
Analyzing the social context present in a gameplay environment and its effect on player experience can provide insights informing the design and social value of games. We investigate the influence of social condition (cooperative or competitive play with a human player versus computer-controlled character) on player experience. The study controlled for co-presence by ensuring that another individual attending to the same stimulus was present in all conditions. Although physiological measures were not significant, subjective measures of arousal and pleasure were significantly different under varying conditions.
\end{abstract}

\section{Author Keywords}

Video Games; Co-located Play; Multiplayer; Single

Player; Physiological Methods; HR; SC; EEG

\section{ACM Classification Keywords}

H.5.m. Information interfaces and presentation (e.g., HCI): Miscellaneous. K.8.0 [Personal Computing]: General -Games

\section{Introduction}

This paper seeks to understand the social context of games and the effects of varying social gameplay conditions. Our main research question is whether social conditions (i.e., social cooperative, competitive, multiplayer environments, or computer-controlled single-player environments) are comparable, or if there 
are conditions that affect the player experience more significantly. However, the presented study extends this concept by consistently having another player in the same room as the participant. Thus, investigators are able to study co-located multiplayer settings and control for the contextual presence of a non-player attending to the game. By comparing these conditions, the paper seeks to investigate whether experiential effects are due to the physical presence of another person, or if self-reported differences are caused by factors in multiplayer interaction with either a

computer-controlled character or human player [11].

\section{Related Work}

Humans are always in a social environment. Previous research links social context to player experience. A recent study reported that playing against a computer can even cause players to be more aggressive than when competing with fellow humans [13]. Emmerich [4] took a qualitative approach to understanding the effects of the presence of friends or strangers on player experience. The paper claims that the social play experience can be affected by a set of factors including the number of players present, the relationship between players (i.e. friends or strangers), the interaction imposed on players by the game itself (e.g. competition or cooperation), the communication mechanism of the game (e.g. chat versus face-to-face communication), and the attendance of players or spectators [5]. One study [13] explores how cooperation and competition affect player experience by creating a game that allows for consistency between game modes to make the variable (social interaction) comparable. Researchers found that cooperation was more likely to inspire empathy, while competition yielded high positive affect. This result is also supported by [12], which demonstrated that the competitive condition has higher affect, as well as promoting the expression of aggression [4]. This illustrates that there is a measurable difference in player experience scores depending on the social context. However, the study relied on self-report data. Both William [13] and Emmerich [4] indicate that an increase in aggression arises in competitive gameplay. Similarly, in a paper comparing the nature of the opponent including a human friend, stranger, or a computer player, significant effects on spatial presence were found [8]. Researchers suggested that this change affects both player arousal and attention. Physiological measures such as EEG can be used to understand player experience by investigating the player's brain state during game events. Using EEG, HFAA can be used to measure a participant's arousal level. A study by Salminen et al. [10] explores how game events modulate player arousal. The study also divides the playing conditions into cooperative and competitive gameplay. The authors use HFAA to investigate the arousal levels of the player. The authors note that more arousing games are perceived as more enjoyable [10]. Wehbe et al. [11] also used HFAA as an indicator of arousal in the context of player experience. Since HFAA is multivalent, the study compared the data to a secondary measure to anchor the results.

\section{Hypotheses}

The first hypothesis examines the differences in social situations and their deviation from the control condition when playing with computer-controlled characters or artificial intelligence (AI). H1: Social playing conditions (competitive, cooperative, and computer-controlled character) will show significant differences in affect (physiological and self-reported arousal, pleasure, and 
dominance). In particular, the literature has illustrated a positive difference in player experience when playing against a human character. Therefore, H2: The competitive and cooperative conditions will show significantly different affect in comparison to the computer-controlled character condition. Finally, H3: The competitive condition will elicit more negative valence in comparison to the cooperative condition.

\section{Methodology}

This study uses a three-level factorial withinparticipants design. Each factor level represents a different social playing condition (cooperative, competitive, and computer-controlled character). In the cooperative condition, the player's character and the confederate's character were on the same team and are competing against two computer-controlled characters. In the competitive condition, the player competes with the confederate. In this condition, the player's character is assisted by one computer-controlled character and faces the confederate, who is assisted by one computer-controlled character. In the computercontrolled character condition, the player competes against two computer-controlled characters, assisted by a computer-controlled character partner. The experimenter acting as a confederate kept social interactions to a minimum (talking, instructing) and only responded to the player briefly if addressed. The confederate set-up the game for the participant, the participant was always aware of the game condition (co-operative, competitive, or computer-controlled). Order of presentation was randomized to reduce learning effects. In all conditions, the confederate is present in the room.
All measures that were used can provide information on the participant's level of arousal: Skin Conductance (SC), Heart Rate (HR), Heart Rate Variability (HRV), and electroencephalography (EEG). The ANT ASA system that was used for the collection of EEG data features 64 channels in a 10-20 layout gel-based electrode cap with a $2048 \mathrm{~Hz}$ sampling rate. The electrodes correspond to the frontal lobe on opposite sides of the head, which are compared in HFAA. The electrodes used are: FP1, AF7, AF3, F7, F5, F3, F1, FT7, $F C 5$, and FC3 in comparison to FP2, AF4, AF8, F2, F4, F6, F8, FC2, FC4, FC6, and FT8. HFAA was used to understand the arousal of the participant $[3,10,12]$. A Nexus II Mark 10 device was used to obtain HR and HRV data using silver electrodes with a $256 \mathrm{~Hz}$ sampling rate. HR was collected by placing sensors on the arms. For the raw data, both HR and HRV were calculated for each condition. The Nexus system was also used to measure skin conductance with a $256 \mathrm{~Hz}$ sampling rate. Electrodes were placed on participants' ring and little fingers. The SC of the participant was used as another measure of arousal in this study. The SAM [8] was used to better understand the participant's perception of dominance, pleasure, and arousal during play.

The game used was Dungeon Duos, a mini-game within Mario Party 4 (Nintendo, 2002). The game was played on the Nintendo Wii with Gamecube controllers. The game features two opposing teams; players must cooperate with their teammate to pass safely through obstacles in the fastest way possible. The fastest team escapes the dungeon and wins the race.

In total, 32 participants were invited to participate in the study. Two participants were excluded because of 


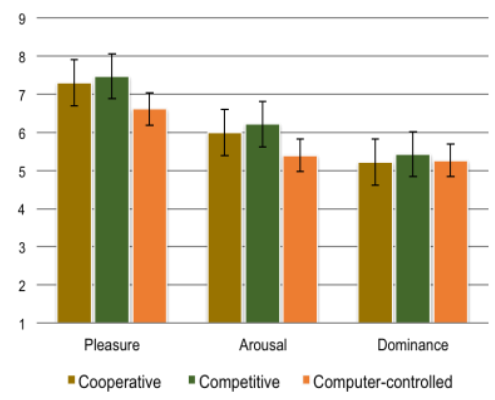

Figure 1: Results of the SAM. The above graph shows the results of SAM dimension compared for each condition. equipment failure, leaving a total of 30 participants: 15 female and 15 male. The average age of participants was 21 years, ranging from 18-34 years. All

participants reported that they have been playing video games for over five years. The final analysis resulted in a total of 23 data sets from participants used in the EEG analysis (seven sets were excluded due to sensor or recording errors)

\section{Results}

A within-participants general linear model repeatedmeasures analysis of variance (RM ANOVA) was conducted for average heart rate (HR) by social condition. The data was tested by Mauchly's test of sphericity. Sphericity $\left(x^{2}(2)=22.952, p=0.001\right)$ was violated, and the RM ANOVA was corrected with a Greenhouse-Geisser (GG) estimate. Therefore, HR was not significantly different between conditions. In other words, all conditions elicited HR that was statistically similar. The HRV data was also analyzed using a RM ANOVA. The data also violated Mauchly's test of sphericity $\left(\mathrm{x}^{2}(2)=39.442, \mathrm{p}<0.001\right)$. Therefore, the ANOVA was calculated using GG. The HRV was not significantly different between the experimental conditions. A within-measures ANOVA was also calculated for SC. The data also violated Mauchly's test of sphericity $\left(x^{2}(2)=107.928, p<0.001\right)$. The ANOVA with GG correction was again not significant. HFAA was calculated from the raw EEG data. The data was exported from the ASA software after using the FFT function to divide the waves into their component frequency bands. Using MATLAB, the electrode data were divided into right and left hemisphere, having the baseline subtracted (as well as logarithmic normalization of the data). The final calculation used was $\ln R-\ln L=\ln (R / L)$ in accordance with $[1,3]$. The data was then analyzed in the IBM SPSS statistics software using a repeated-measures general linear model. The data for three different social playing conditions (computer-controlled, cooperative, and competitive) were calculated using HFAA. Mauchly's test of sphericity was not violated and sphericity was assumed. The test of within-participants effects with sphericity assumed was not significant. For the SAM data, pleasure was run with a RM ANOVA. Mauchly's test of sphericity was not significant. The ANOVA resulted in $F(2,44)=3.021, p=0.059, \eta_{p}{ }^{2}=0.121$. Therefore, the pleasure dimension was not significantly different between conditions. Pairwise comparisons reveal that there is no significant difference between cooperative and single-player gameplay with computercontrolled players $(p=0.054)$ or cooperative and competitive $(p=0.698)$. However, there were significant differences in pleasure ratings for competitive versus single-player (playing with computer-controlled characters) conditions $(p=0.015)$. Arousal data was tested for significant differences using an RM ANOVA Mauchly's test of sphericity was significant $\left(X^{2}(2)=\right.$ $6.827, \mathrm{p}=0.033)$. The RM ANOVA was also significant $F(1.566,34.441)=3.847, p=0.040, \eta_{p}^{2}=0.149$. Pairwise comparisons reveal significant differences in arousal between multiplayer competitive, and singleplayer (computer-controlled) conditions $(p=0.039)$. Additional arousal differences were found between cooperative play and single player computer-controlled players $(p=0.016)$. As Figure 1 shows, both cooperative and competitive gameplay scored higher in arousal than computer-controlled gameplay. However, pairwise comparison reveals no significant differences between playing cooperatively or competitively when co-located with another player $(p=0.478)$. The dominance data was analyzed with a RM ANOVA. Neither Mauchly's test 
of sphericity nor the RM ANOVA were significant. Therefore, there is no significant difference between conditions and the feeling of dominance.

\section{Discussion}

Literature in the field has demonstrated significant differences in measures such as self-reported enjoyment and aggression when playing with a person versus playing with a computer-controlled character $[4,13]$. We sought to demonstrate this difference objectively using physiological measures, but did not succeed. Significant differences between social gameplay conditions were not found using physiological measures (EEG, HR, HRV, SC) in this study. However, the SAM questionnaire does indicate some potential differences in user experience. Pleasure ratings on the SAM reveal that players felt more pleasure when competing against a person. However, the same cannot be said about cooperation. Although the results reveal a trend, cooperating with a computer versus a human player did not significantly affect the level of pleasure experienced by the player. The least significant difference was found between cooperative and competitive play with humans. Additionally, there were significant differences in perceived arousal between social gameplay conditions. Significant differences were found for both cooperative play versus single-player AI and competitive play versus single-player AI. However, significant differences were not found between playing cooperatively or competitively with another person.

The player rated their enjoyment of the game differently based on the participation of the confederate, despite the minimal time difference between rounds. These findings suggest that although the player may have just played with the confederate the round before, there is a significant drop in arousal when the confederate withdraws from the match.

The study seeks to control for co-presence such that effects observed stem from the type of social interaction. In addition, the literature supports that the relationship to the person with whom the player is interacting impacts the player [9]. Further research is thus needed to fully understand how the effects of copresence and social relationships interact to influence the player experience. Follow-up studies are needed to determine if the players' arousal level depends on their perception of fellow players, or the actual nature of those players.

Future studies will also look at non-co-located conditions to understand the interactions between variables. Therefore, the following is left as a question for future research: How do the effects of co-located individuals differ when participants are mutually present in the same platform or community group?

\section{Future Work}

Overall results of the study are surprising, considering the strong relationship presented by the literature in the field. Possibly, the results could be attributed to either the general fun factor of the Mario Party minigame (everything is equally exciting) or the use of an easy difficulty in the study (without a significant challenge from the computer, we do not witness arousal stemming from possible frustration). On the other hand, each condition includes computercontrolled characters in some cooperative or competitive form, which might result in evening out any skill-based discrepancies between players that we might have been able to witness otherwise. Future 
studies may seek to test these variables. This study sought to understand a player's level of arousal as a primary measure of player experience. This is in accordance with the literature, which studies arousal as an indicator of excitement $[2,7,10]$. However, all permutations of the environment, game choice, and study design are not explored. The study conducted in this paper only explores conditions where the human confederate was seated beside the player and did not explore other conditions of social play, such as playing online. Future work may also incorporate research on the effects of observation on player experience [6].

\section{Acknowledgements}

We would like to thank SSHRC, NSERC, and GRAND NCE for their support. Additionally, Rina R. Wehbe would like to thank OGS and University of Ontario Institute of Technology (UOIT) for their support. This work was presented as part of Rina R. Wehbe's M.Sc. Computer Science thesis at UOIT, Canada. We would also like to thank Abdulaziz Almehmadi, Jens Johannsmeier, and Samantha Stahlke for their support.

\section{References}

1. Allen, J.J.B., Coan, J. a, and Nazarian, M.Issues and assumptions on the road from raw signals to metrics of frontal EEG asymmetry in emotion. Biological psychology 67, 1-2 (2004), 183-218.

2. Chanel, G., Kivikangas, J.M., and Ravaja

N.Physiological compliance for social gaming analysis: Cooperative versus competitive play. Interacting with Computers 24, 4 (2012), 306-316.

3. Coan, J. a and Allen, J.J.B.Frontal EEG asymmetry as a moderator and mediator of emotion. Biological psychology 67, 1-2 (2004), 7-49.

4. Emmerich, K. and Masuch, M. Helping Friends or Fighting Foes: The Influence of Collaboration and Competition on Player Experience. 8th International
Conference on the Foundations of Digital Games, (2013).

5. Kappen, D.L., Gregory, J., Stepchenko, D., Wehbe, R.R., and Nacke, L.E.Exploring social interaction in colocated multiplayer games. CHI '13 Extended Abstracts on Human Factors in Computing Systems on - CHI EA '13, (2013), 1119.

6. Kappen, D.L., Mirza-Babaei, P., Johannsmeier, J., Buckstein, D., Robb, J., and Nacke, L.E.Engaged by boos and cheers. Proceedings of the first ACM SIGCHI annual symposium on Computer-human interaction in play - CHI PLAY'14, (2014), 151-160.

7. Kivikangas, J., Chanel, G., Cowley, B., et al.A review of the use of psychophysiological methods in game research. Proc. Nordic DiGRA'10 3, 3 (2011), 181-199.

8. Lang, P.J.Behavioral treatment and bio-behavioral assessment: Computer applications. Technology in Mental Health Care Delivery Systems. Ablex Pub. Corp. Norwood, New Jersey, USA, New Jersey, USA, 1980.

9. Ravaja, N., Saari, T., and Turpeinen, M.Spatial presence and emotions during video game playing: Does it matter with whom you play? Presence, (2006), 327-333.

10.Salminen, M.J., Kivikangas, M., Ravaja, N., and Kallinen, K.Frontal EEG Asymmetry in the Study of Player Experiences during Competitive and Cooperative Play. Proceedings of the IADIS International Conference on Game and Entertainment Technologies,

International Association for Development of the Information Society (2009), 44-50.

11.Stenros, J., Paavilainen, J., and Mäyrä, F.The many faces of sociability and social play in games.

Proceedings of the 13th International MindTrek Conference: Everyday Life in the Ubiquitous Era on MindTrek '09, (2009), 82.

12. Wehbe, R.R., Kappen, D., Rojas, D., Klauser, M., Kapralos, B., and Nacke, L.E.EEG-based assessment of video and in-game learning. Proc. $\mathrm{CHI}^{\prime} 13$ Extended Abstracts, (2013).

13. Williams, R.B. and Clippinger, C. a.Aggression, competition and computer games: computer and human opponents. Computers in Human Behavior 18, 5 (2002), 495-506. 